\title{
ESSENTIAL SPECTRA OF QUASI-PARABOLIC COMPOSITION OPERATORS ON HARDY SPACES OF THE POLY-DISC
}

\author{
UĞUR GÜL
}

Abstract. In this paper we study the essential spectra of a class of composition operators on the Hilbert-Hardy space of the bi-disc which is called "quasi-parabolic" and whose one variable analogue was studied in [2]. As in [2], quasi-parabolic composition operators on the Hilbert-Hardy space of the bi-disc are written as a linear combination of Toeplitz operators and Fourier multipliers. The $\mathrm{C}^{*}$-algebra generated by Toeplitz operators and Fourier multipliers on the Hilbert-Hardy space of the bi-disc is written as the tensor product of the similar $\mathrm{C}^{*}$-algebra in one variable with itself. As a result we find a nontrivial set consisting of spiral curves lying inside the essential spectra of quasi-parabolic composition operators.

Mathematics subject classification (2010): 32A45.

Keywords and phrases: Composition operators, Hardy spaces of the poly-disc, essential spectra.

\section{REFERENCES}

[1] R. G. Douglas, R. Howe, On the $C^{*}$-algebra of Toeplitz operators on the quarterplane, Trans. Amer. Math. Soc., 158 (1971), pp. 203-217.

[2] U. GüL, Essential Spectra of Quasi-parabolic Composition Operators on Hardy Spaces of Analytic Functions, J. Math. Anal. Appl., 377 (2011), pp. 771-791.

[3] K. Hoffman, Banach Spaces of Analytic Functions, Prentice-Hall Inc., Englewood Cliffs, N.J., 1962.

[4] F. JAFARI, On Bounded and Compact Composition Operators in Poly-discs, Canadian J. Math. 42 (1990), pp. 869-889.

[5] G. Murphy, C*-algebras and Operator Theory, Academic Press Inc., 1990.

[6] W. Rudin, Functional Analysis, McGraw Hill Inc., 1973.

[7] J. H. Shapiro, Cluster set, essential range, and distance estimates in BMO, Michigan Math. J. 34 (1987), no. 3, 323-336.

[8] H. UpmeIER, Toeplitz Operators and Index Theory in Several Complex Variables, Operator Theory Advances and Applications vol. 81, Birkhäuser, 1996. 